Sunyaev: The best wavelength for observations of "real" cosmological fluctuations is the band close to the maximum of the $2.7 \mathrm{~K} \mathrm{black}$ body spectrum. However, the atmospheric conditions are better in the centimetre band. The best angular scales are indicated in the written version of this paper.

\title{
CONSTRAINTS ON THE MEAN DENSITY OF THE UNIVERSE WHICH FOLLOW FROM THE THEORIES OF ADIABATIC AND WHIRL PERTURBATIONS
}

\section{A. A. Kurskov and L. M. Ozernoy}

The aim of this communication is to investigate what constraints to the cosmological parameter $\Omega=2 q_{0}$ can be obtained if one assumes that primaeval whirl motions or adiabatic density perturbations with an appropriate initial spectrum were responsible for the formation of large scale structure in the Universe. These constraints are readily obtained from the two conditions: (i) an upper limit to small scale temperature fluctuations of the microwave background radiation, and (ii) the requirement that the primaeval perturbations should be large enough in order to produce observed structures.

(a) Adiabatic perturbations. One usually makes the reasonable assumption that the initial metric perturbations are scale-independent: $\mathrm{h}=\mathrm{h}_{\mathrm{i}}=$ constant ("white noise"). Then using Chibisov's (1972) value for the mass $M_{d}$ damped at decoupling we are able to show (Kurskov and Ozernoy 1977a) that the condition of isolation $\left(\Delta \rho / \rho \simeq 1\right.$ at $\left.z \simeq \Omega^{-1}\right)$ for inhomogeneities of maximum amplitude corresponding to masses $M \sim M_{d}$ is $\mathrm{h}_{\mathrm{i}}>2 \times 10^{-4} \Omega^{-5 / 4} \mathrm{~h}^{-1 / 2}\left(1+-3 \Omega \mathrm{h}^{2}\right)$, where $\mathrm{h}=\mathrm{H}_{\mathrm{O}} / 75 \mathrm{~km} \mathrm{~s}^{-1} \mathrm{Mpc}^{-1}$. Since the r.m.s. temperature fluctuations produced at the epoch of recombination due to the Doppler-effect on potential velocities (Zeldovich and Sunyaev 1970$)$ are $\Delta \mathrm{T} / \mathrm{T} \sim(\sqrt{3})^{-1}\left(\mathrm{v}_{\mathrm{rec}} / \mathrm{c}\right)=1 / 3\left(\mathrm{z}_{\mathrm{rec}} / \mathrm{z}_{\mathrm{eq}}\right)^{3 / 4}$ $h_{i}$ on characteristic mass-scale $M \leqslant M_{J}\left(t_{r e c}\right)$, one finds for $0.5<\Omega \mathrm{h}^{2}<0.7$ that $\Delta \mathrm{T} / \mathrm{T} \geqslant 10^{-5} \Omega^{-2} \mathrm{~h}^{-2}\left(1+3 \Omega \mathrm{h}^{2}\right)$ on angular scales $\theta \leqslant 15^{\prime}-30^{\prime}$. Comparison with corresponding upper limit $\Delta \mathrm{T} / \mathrm{T} \leqslant 10^{-4}$ yields $\Omega \geqslant 0.3\left[(\Delta \mathrm{T} / \mathrm{T}) / 10^{-4}\right]^{-1}$. This conclusion cannot be avoided by introducing secondary reheating since otherwise appreciable angular variations of $\Delta \mathrm{T} / \mathrm{T} \geqslant 10^{-3}$ on scales $\theta \sim 20^{\circ} \Omega^{2 / 3}$ will appear (Kurskov and Ozernoy 1977a) unless the initial metric perturbation spectrum had a cut-off on masses $\mathrm{M} \ll 10^{22} \mathrm{M}_{\odot}$ which seems to be rather artificial.

(b) Whirl perturbations. Angular variations of $\Delta \mathrm{T} / \mathrm{T}$ produced by the Doppler-effect on turbulent velocities (Chibisov and Ozernoy 1969) are the sum of (i) temperature variations at $t=t_{\text {rec }}$ weakened by Thompson scattering due to secondary reheating of the cosmic plasma by young galaxies and of (ii) temperature variations produced at the moment of "last scattering" when $\tau \approx 1$. The latter gives the main contri-

*This lower limit to $\Omega$ is apparently even more stringent if $\Omega \mathrm{h}^{2} \gg 0.1$ since in that case appreciable velocities are present on scales $M>M_{J}\left(t_{\text {rec }}\right)$ where oscillations were always absent. 
bution to the observed value of $\Delta \mathrm{T} / \mathrm{T}$ at comparatively large $\mathrm{W}$ $\left(\mathrm{W} \geqslant 0.24-0.31\right.$ when $\left.\Omega \mathrm{h}^{2}=1-0.1\right)$ which are of most importance in the whirl cosmogony.* Comparison with the observed upper limit $\Delta \mathrm{T} / \mathrm{T} \leqslant 10^{-4}$ shows that the whirl theory in its present state is invalid unless $\Omega \mathrm{h}^{2} \geqslant 0.3-0.4$.

We conclude that both primaeval whirls or "white noise" adiabatic density perturbations responsible for galaxy formation may be compatible with the available upper limits to $\Delta \mathrm{T} / \mathrm{T}$ only in a dense enough Universe $\left(\Omega h^{2} \geqslant 0.3-0.4\right)$.

\section{REFERENCES}

Anile, A.M., Danese, L., De Zotti, G. and Motta, S., 1976. Astrophys.J., Chibisov, G.V., 1972. Astron. Zh., 49, 74.

Chibisov, G.V. and Ozernoy, L.M., 1969. Astrophys. Lttters, 3, 189. Kurskov, A.A. and Ozernoy, L.M., 1977a. Astrophys. Sp. Sci. (submitted). Kurskov, A.A. and Ozernoy, L.M., 1977b. Astrophys. Sp. Sci. (submitted). Zeldovich, Ya. B. and Sunyaev, R.A., 1972. Astrophys. Sp. Sci., $\underline{7}, 3$.

*It should be noted that the value of $\Delta \mathrm{T} / \mathrm{T}$ from the last scattering calculated by Anile et al. (1976) and used by Parijskij (this meeting) was overestimated by about one order of magnitude (Kurskov and Ozernoy 1977b).

\section{RECENT ADVANCES IN MICROWAVE COSMOLOGY}

\section{Paul Boynton}

In summary, this brief communication outlined first the recent measurement of large-scale anisotropy of the relic radiation by the Berkeley group of Smoot, Gorenstein and Muller (1977). The customary peculiar velocity interpretation of the dipole term gives $v=390 \pm 60$ $\mathrm{km} / \mathrm{sec}$ in the direction of $\alpha=11.0 \pm 0.5$ and $\delta=6^{\circ} \pm 10^{\circ}$. These values differ from the preliminary results of Corey and Wilkinson (1976) by less than twice their reported errors.

Secondly, the latest observations by G. Lake and R. B. Partridge (1977a, 1977b) of clusters of galaxies seeking evidence for inverse Compton "cooling" of the microwave background were reviewed. Significant effect was indicated in each of the richness class 4 clusters observed:

\begin{tabular}{cc} 
Abe11 Cluster & $\Delta \mathrm{T} \pm 1 \sigma$ \\
\hline 1689 & $-1.06 \pm 0.46 \mathrm{mK}$ \\
2125 & $-3.10 \pm 0.34 \mathrm{mK}$ \\
2218 & $-2.65 \pm 0.23 \mathrm{mK}$ \\
\hline
\end{tabular}

